\title{
L'économie de fonctionnalité : principes, éléments de terminologie et proposition de typologie.
}

The functional service economy: definition, principles, terminology, typology and some examples.

Johan Van Niel

\section{OpenEdition}

\section{Journals}

Édition électronique

URL : https://journals.openedition.org/developpementdurable/10160

DOI : 10.4000/developpementdurable.10160

ISSN : 1772-9971

Éditeur

Association DD\&T

Référence électronique

Johan Van Niel, «L'économie de fonctionnalité : principes, éléments de terminologie et proposition de typologie. », Développement durable et territoires [En ligne], Vol. 5, n¹ | Février 2014, mis en ligne le 04 février 2014, consulté le 21 septembre 2021. URL : http://journals.openedition.org/ developpementdurable/10160 ; DOI : https://doi.org/10.4000/developpementdurable.10160

Ce document a été généré automatiquement le 21 septembre 2021.

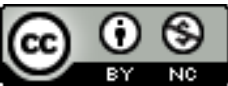

Développement Durable et Territoires est mis à disposition selon les termes de la licence Creative Commons Attribution - Pas d'Utilisation Commerciale 4.0 International. 


\section{L'économie de fonctionnalité : principes, éléments de terminologie et proposition de typologie.}

The functional service economy: definition, principles, terminology, typology

and some examples.

Johan Van Niel

1 L'économie de fonctionnalité consiste en « la substitution de la vente de l'usage d'un bien à la vente du bien lui-même» (Bourg et Buclet, 2005 : 2). La reproduction de document avec paiement «à la copie » de la société Xerox est certainement l'un des exemples les plus connus en matière d'opérationnalisation réussie de ce concept. Ce n'en est cependant qu'une de ses nombreuses déclinaisons.

2 La première partie de cet article sera consacrée à un bref retour sur les origines et les bases théoriques de l'économie de fonctionnalité, puis s'intéressera à l'évolution de la terminologie employée dans différents travaux de recherche anglophones pour désigner certaines combinaisons de produits et de services, dont il sera montré qu'elles consistent, au moins en partie, en des concepts directement apparentés à l'économie de fonctionnalité.

3 S'inspirant de la diversité de catégories d'offres de solutions qu'il aura ainsi été possible d'identifier, la seconde partie de l'article proposera une typologie destinée à caractériser les grandes catégories de modèles de revenu auxquels peuvent recourir les entreprises en vue de déployer ce modèle économique et s'efforcera de montrer, à partir d'une description de leurs mécanismes et d'une sélection d'études de cas, en quoi ceux-ci sont susceptibles de présenter des avantages, d'un point de vue environnemental, par rapport à la vente de produits.

4 Pour clore cette énumération des modes de mise en application pratique des principes de l'économie de fonctionnalité, la dernière partie de l'article s'efforcera de montrer que ceux-ci ne se limitent pas nécessairement à la sphère du marché, mais peuvent également 
prendre la forme d'échange reposant sur la mutualisation de biens et services entre individus, y compris au travers de systèmes de transactions non marchandes tels que les Systèmes d'Échanges Locaux. Ceci débouchera sur une proposition de définition visant à élargir la compréhension que l'on peut avoir de son domaine d'application.

\section{L'économie de fonctionnalité : principes et terminologie}

5 Les origines du concept d'économie de fonctionnalité remontent au début des années 1980, alors que commençait à émerger la vision d'une économie circulaire dans les milieux scientifiques qui relayaient les idées du Club de Rome. En vertu du fait qu'il est moins coûteux d'un point de vue énergétique de rénover du matériel que de recycler des molécules, Walter Stahel faisait partie de ceux qui défendaient l'idée qu'une stratégie visant à optimiser l'éco-efficacité des produits ne doit pas se limiter à des démarches « end of pipe » axées sur l'extension de leur durée de vie, la prévention des déchets, ou encore le recyclage des matériaux, mais doit avant tout mettre l'accent sur le bouclage de leur cycle de vie. Il est ainsi selon lui nécessaire de migrer d'une approche qui n'envisage l'optimisation des flux de matières et d'énergie que «du berceau jusqu'à la tombe »comprendre : de la carrière à la décharge - à une approche où la vie des produits serait étendue "du berceau jusqu'au nouveau berceau », autrement dit passer d'un système industriel linéaire à une « économie circulaire ». Le modèle économique le mieux adapté à cette fin, développait-il, est celui où c'est l'usage du produit qui est vendu, et non le produit lui-même (Stahel et Giarini, 1989).

\subsection{Bases théoriques}

«L'économie de fonctionnalité, qui optimise l'usage ou la fonction des biens et services, se concentre sur la gestion des richesses existantes, sous la forme de biens, de connaissances et de capital naturel. L'objectif économique en est de créer une valeur d'usage la plus élevée possible pendant le plus longtemps possible, tout en consommant le moins de ressources matérielles et d'énergie possible » (Stahel, 2006 : 145). En effet, dans un modèle économique où l'objet de la transaction n'est plus le transfert de la propriété d'un bien matériel, mais la fonction qu'il remplit - c'est-à-dire où, concrètement, on vend « de la mobilité plutôt qu'un véhicule, des services de nettoyage au lieu de lessives, des films et non des vidéocassettes " (Mont, 2002 : 140) - le rapport du producteur à ses produits est radicalement modifié.

7 Les produits n'apparaissent dans une telle perspective plus que comme de simples moyens, comme des intermédiaires dans un processus visant à délivrer une fonction, au lieu d'être l'objet central de la négociation. Puisque c'est désormais lui qui en conserve la propriété (et donc la responsabilité), ils deviennent donc une forme de capital que le producteur a tout intérêt à gérer sérieusement et efficacement. Leur valeur économique ne résidant plus dans leur valeur d'échange, mais dans leur valeur d'usage, il va devoir s'intéresser non plus seulement à leur prix de revient (c'est-à-dire les coûts associés à leur conception et leur fabrication), mais au coût global du service rendu, qui prend également en compte leurs phases de fonctionnement, de maintenance ou encore de traitement en fin de vie. Et puisque c'est désormais lui qui supporte ces coûts et qui est pénalisé en cas de panne, il va ainsi devoir abandonner l'approche industrielle traditionnelle, qui repose sur la production en masse de produits à obsolescence 
programmée, et s'orienter vers des modes de conception et de production qui maximisent la fiabilité, la durée de vie opérationnelle et la sobriété de ces supports fonctionnels.

8 Sa rentabilité économique ne dépendant plus de la maximisation de la quantité de produits qu'il vend mais du nombre d'« unités fonctionnelles» que le producteur est capable de fournir à ses clients à partir d'une quantité de capital donné, ces "supports fonctionnels " doivent donc être conçus de façon à être modulables et facilement démontables, dans le but de faciliter la rénovation, l'éventuelle amélioration, la réutilisation ou le recyclage de leurs différents composants et matériaux. Les gains retirés de l'emploi répété et prolongé d'un même support fonctionnel permettant de rendre acceptable une augmentation de son coût de revient, il devient possible de consacrer davantage de temps et de moyens à l'écoconception de celui-ci, en définissant des coefficients de sécurité plus élevés, en choisissant des matériaux plus robustes, aisément recyclables et moins nocifs pour l'environnement, etc.

Par un simple changement de perspective, on passe donc d'une rationalité économique imposant la maximisation de la production et la consommation de produits volontairement déficients à une rationalité qui préconise au contraire l'augmentation de la durée de circulation des supports fonctionnels dans le processus économique, le bouclage de leur cycle de vie et l'optimisation de leur taux d'utilisation et leur écoefficacité. C'est ainsi à partir du constat que sa mise en œuvre permet d'amorcer un certain découplage entre création de valeur économique et pressions environnementales que Walter Stahel est capable d'affirmer que "l'économie de fonctionnalité est considérablement plus soutenable, ou dématérialisée, que l'économie actuelle, qui se concentre sur la production comme principal moyen de créer de la richesse et des flux de ressources " (Stahel, 1997 : 91).

Notons que, en plus de modifier le rapport du producteur à ses produits, le passage à une économie de fonctionnalité a pour effet de changer les relations entre acteurs de l'échange. Au lieu d'être antagonistes, leurs intérêts tendent en effet désormais au contraire à converger : «dans le schéma de pensée économique traditionnel, les producteurs sont considérés comme créateurs de valeur et les clients comme destructeurs de valeur. Dans une économie de fonctionnalité, les producteurs deviennent fournisseurs de valeur et les clients utilisateurs de valeur. Il devient de l'intérêt des deux parties de faire en sorte que la fonction soit constamment remplie et que la valeur soit continuellement fournie » (Mont, $2004: 30$ ). On passe donc d'une situation de compétition entre producteur et consommateur autour du prix de vente du produit - l'un cherchant à vendre au prix le plus élevé possible, et l'autre à acheter au prix le plus bas possible - à des relations, généralement contractuelles, dans lesquelles le prestataire va chercher à satisfaire au mieux les besoins de son client, allant même dans certains cas jusqu'à coproduire avec lui la fonction.

\section{2. Éléments de terminologie anglophones et francophones}

11 Dans l'ouvrage internationalement reconnu comme fondateur du concept d'économie de fonctionnalité, Walter Stahel employait pour désigner celui-ci l'expression "new service economy" (Stahel et Giarini, 1989). Prêtant de toute évidence à confusion, cette appellation connaîtra par la suite plusieurs évolutions destinées à mettre davantage l'accent sur ce qui le distingue du courant dominant de l'économie des services. Dans ses travaux ultérieurs, Walter Stahel emploiera ainsi des termes faisant apparaître plus explicitement les notions d'usage et de fonction: "Utilization-Focused Service Economy" (Stahel, 1994), "F 
unctional Economy" (Stahel, 1997), "Functional Service Economy" (Stahel, 2006). Notons qu'il est important de ne pas confondre le concept d'économie de fonctionnalité avec le concept plus englobant de "Performance Economy", qu'il développe dans son ouvrage le plus récent. L'économie de fonctionnalité, en tant que modèle économique alternatif au modèle dominant, n'y est présentée que comme l'un des rouages - au même titre que les stratégies d'écoconception, par exemple - d'une société qui serait centrée sur le bouclage des flux.

L'idée d'une économie où l'accès à l'usage des produits viendrait supplanter le transfert de leur propriété fit l'objet de plusieurs programmes de recherche, en Europe et aux États-Unis notamment, au cours des deux dernières décennies. Il en résulta une grande diversité de notions et de définitions (cf. Tableau 1).

Tableau 1 : Notions anglophones apparentées au concept d'économie de fonctionnalité

\begin{tabular}{|c|c|}
\hline $\begin{array}{l}\text { Eco-efficient } \\
\text { services }\end{array}$ & $\begin{array}{l}\text { "systèmes de produits et services qui sont développés de telle façon à causer un } \\
\text { minimum d'impact environnemental pour un maximum de valeur ajoutée " } \\
\text { (Brezet et al., 2001:8). }\end{array}$ \\
\hline $\begin{array}{l}\text { Eco-efficient } \\
\text { producer services }\end{array}$ & $\begin{array}{l}\text { "un certain mélange de produits et de services qui a une plus grande valeur } \\
\text { ajoutée et un plus faible impact environnemental comparé à un mélange de } \\
\text { produits et de services similaire ou à une situation où l'activité n'avait pas du tout } \\
\text { lieu» (Zaring et al., 2001:37). }\end{array}$ \\
\hline Servicizing & $\begin{array}{l}\text { "l'émergence de services fondés sur des produits, qui brouillent la distinction entre } \\
\text { les activités de fabrication et celles du secteur des services traditionnels " (White } \\
\text { et al., 1999: 10). }\end{array}$ \\
\hline Functional sales & $\begin{array}{l}\text { "offrir dans une perspective de cycle de vie une solution fonctionnelle permettant } \\
\text { de satisfaire un besoin donné du consommateur. (...) La solution fonctionnelle peut } \\
\text { être constituée d'une combinaison de systèmes, d'objets et de services" (Lindahl et } \\
\text { Ölundh, } 2001: 6)^{1} \text {. }\end{array}$ \\
\hline $\begin{array}{l}\text { Product-Service } \\
\text { Systems (PSS) }\end{array}$ & $\begin{array}{l}\text { "un PSS est constitué de produits tangibles et de services intangibles conçus et } \\
\text { combinés de façon à être capables de satisfaire conjointement les besoins } \\
\text { spécifiques des consommateurs» (Tukker et al., 2004:17) }{ }^{2} \text {. }\end{array}$ \\
\hline
\end{tabular}

De nombreux autres articles et rapports ont bien entendu été publiés en parallèle ou en dehors du cadre des travaux que nous venons d'identifier. L'objectif n'est pas ici d'essayer d'en dresser une revue exhaustive, mais de montrer à partir des exemples que nous considérons comme les plus significatifs la grande diversité de la terminologie employée pour désigner des concepts pourtant très apparentés à celui de Walter Stahel. Il suffit pour se convaincre de l'existence de ce lien de parenté direct de comparer leurs différentes définitions, et au cas où cela ne serait pas une preuve suffisante, de constater que ces différents travaux s'appuient sur des études de cas la plupart du temps similaires, qu'ils s'efforcent d'ailleurs d'analyser en fonction de critères semblables (potentiel de dématérialisation, forces et barrières...). Cette prolifération de termes semble s'être arrêtée avec l'arrivée du concept-phare de Product-Service Systems, autour duquel se cristallise depuis les premières années du nouveau millénaire la production de littérature scientifique s'intéressant aux applications dérivées du modèle de Walter Stahel. De 
nombreux chercheurs se sont là aussi penchés sur le concept, à titre individuel ou dans le cadre d'une nouvelle génération de programmes de recherche. Une revue de la littérature récente, qui recense 149 publications scientifiques portant sur les PSS sur la période allant de 2006 à 2010, montre que les recherches sur le sujet restent essentiellement dominées par des travaux théoriques (Beuren et al., 2013), dont l'objectif est pour l'essentiel de contribuer à la conception d'outils et méthodologies destinés à aider les entreprises à développer de telles offres.

Un phénomène de diversification du vocabulaire de la fonctionnalité semble être également à l'œuvre au sein des milieux universitaires et associatifs francophones. S'il reste pour le moment encore le plus employé, le terme de "fonctionnalité » (employé avec ou sans pronom, au pluriel ou au singulier) ne fait en effet pas l'unanimité, et il n'est guère de séances de travail ou de réflexion autour du concept où n'ait été soulevé la question de l'adoption d'un titre moins abscons, d'une appellation plus simple et plus explicite qui soit plus à même de marquer les esprits et par là de favoriser la diffusion de la notion. Outre l'expression "économie des effets utiles", que semble pour sa part préférer Philippe Moati (2009), on peut ainsi voir fleurir au gré des écrits ou des discussions informelles dont le concept fait l'objet des formules telles que : «économie fonctionnelle " (probablement en référence à l'analyse fonctionnelle, méthodologie qui consiste à envisager un produit sous l'angle des fonctions qu'il permet de remplir), « économie de la fonction » ou encore " économie de l'usage » - cette dernière tendant à être employée « de plus en plus souvent ", selon le magazine Alternatives économiques ${ }^{3}$. Nous avons également identifié l'émergence récente de l'expression «écofonctionnalité ", expression qui permet certes de gagner en concision mais tend surtout selon nous à être source de confusion, le préfixe "éco-» risquant d'être compris davantage comme une contraction de l'adjectif "écologique» que comme une abréviation du mot « économie »".

Cette profusion de termes peut indéniablement être interprétée comme un signe positif, celui d'un intérêt croissant pour le concept et de son appropriation par des acteurs qui y reconnaissent une stratégie permettant de donner corps à un développement qui soit plus soutenable. Elle n'est cependant pas sans danger. Bien que les notions d'usage ou d'effets utiles viennent sans aucun doute enrichir la sphère de sens de l'économie de fonctionnalité, il est à craindre qu'une telle prolifération de termes finisse par générer de la confusion (parle-t-on bien de la même chose ou, s'il y en a, quelles sont les différences fondamentales avec le concept de Walter Stahel?), voire d'aboutir à une dilution progressive du sens du concept original. «La terminologie marketing doit être utilisée avec des pincettes ", nous prévient Walter Stahel: "des concepts tels que servicizing et Product-Service Systems n'incluent pas nécessairement une internalisation par les acteurs économiques des responsabilités et des coûts des déchets. (...) Le terme PSS est devenu populaire au début du troisième millénaire mais dans la plupart des cas passe à côté des problématiques de la performance et de la responsabilité, qui sont des éléments clés de l'économie de fonctionnalité. " (Stahel, 2006 : 194). Nombre de PSS ne répondent en effet pas aux critères de l'économie de fonctionnalité (voir plus bas) et à vouloir recouvrir un éventail trop vaste d'applications pratiques, ce concept court le risque d'endommager sa crédibilité5. Il serait ainsi à notre sens dommageable que la perpétuation de ce phénomène de diversification du champ lexical francophone de la fonctionnalité débouche sur des concepts qui, voulant trop en dire (ou simplement dire autrement) finiraient par ne plus rien vouloir dire. 
Outre une diversité de définitions, les travaux sur les PSS ont débouché sur plusieurs typologies d'offres dont nous nous sommes en partie inspirés pour construire celle que nous présentons dans la partie suivante.

\section{Proposition de typologie}

L'exemple de la location est généralement celui qui vient en premier à l'esprit la première fois qu'on entend parler d'économie de fonctionnalité. C'est aussi, trop souvent, l'arbre qui finit par cacher la forêt. En effet, de même que le capitalisme de marché n'est qu'un système économique bien particulier parmi d'autres, il existe de nombreux modes de transfert entre individus alternatifs à la vente de produits. La satisfaction de nombre de besoins ne requiert ainsi pas nécessairement l'appropriation privative de biens matériels mais peut être obtenue au travers de la possession temporaire de produits ou de prestations de services plus ou moins immatérielles. Dans une logique de fonctionnalité, énonce Christian du Tertre, "l'échange marchand ne relève plus de paiements à l'unité ou à l'acte, mais de paiements à l'accès adossés à l'obtention de résultats en matière de performance qui sont en capacité d'intégrer des objectifs relevant du développement durable » (Du Tertre, 2011 : 35). On ne vend plus un produit, mais l'accès à des fonctions d'usage. Notons au passage que l'économie de fonctionnalité constitue en ces termes une manifestation directe du passage de nos sociétés d'un régime de propriété à un régime d'accès (Rifkin, 2000).

Les relations marchandes, c'est-à-dire les modes de passation de marché avec les fournisseurs et les clients, sont l'une des dimensions d'un modèle économique d'entreprise, que Christian du Tertre (2011:22) identifie au nombre de quatre, les autres étant les modes de production et d'appropriation de la valeur, les modes de mobilisation des ressources humaines et de prise en considération du travail et les modes de financement des investissements matériels et immatériels. On retrouve répertoriés ici les principales composantes d'un «business model » (en français «modèle d'affaires » ou " modèle économique d'entreprise »), dont il existe selon les spécialistes issus du monde académique une profusion de définitions et de typologies. Leur traduction dans le langage des affaires pourrait être, pour les trois dernières : le réseau, la chaine ou l'architecture de valeur, l'organisation des ressources et compétences de l'entreprise, et les charges de l'entreprise. L'organisation des transactions marchandes de l'entreprise correspond pour sa part au «modèle de revenu» de l'entreprise - que Xavier Lecocq et ses collègues considèrent pour leur part sous l'angle du volume et de la structure des revenus générés, d'une part, et des modalités de rémunération de l'entreprise, de l'autre (Lecocq et al., 2006 : 13-14) - autrement dit la façon dont elle gagne de l'argent, typiquement en vendant des produits et services (son offre, ou "proposition de valeur ») à ses clients. C'est en dressant une typologie de leurs modèles de revenu que nous nous proposons de catégoriser les différents modèles économiques d'entreprise qui sont susceptibles d'obéir, au moins en partie, aux principes de l'économie de fonctionnalité.

19 Nous avons choisi de nous inspirer pour cela en partie des typologies de Product-Service Systems que l'on trouve dans la littérature anglophone. En partie seulement parce que, bien qu'ils soient par bien des côtés fortement apparentés, les concepts d'économie de fonctionnalité et de PSS ne sont pas totalement identiques. De fait, ce que l'on range sous le terme englobant de PSS regroupe une compilation assez vaste de différents «complexes de biens et de services liés ou complémentaires", pour reprendre une expression de Jean Gadrey (2003: 48), dont seule une fraction présente des caractéristiques compatibles avec 
les principes de l'économie de fonctionnalité telle que nous nous sommes efforcés de la présenter.

L'analyse des caractéristiques des offres de solutions qu'a réalisée Philippe Moati dans son ouvrage sur "l'économie des bouquets» (Moati, 2008) permet de nous aider à identifier ce qui autorise un PSS à se réclamer ou non de l'économie de fonctionnalité. Selon ses critères d'analyse, tous les PSS sont caractérisés, entre autres mais notamment, par un important degré de complétude, c'est-à-dire un degré de traitement de la fonction fondatrice élevé (Moati, 2008: 27). Or, pour répondre au moins en partie aux critères de l'économie de fonctionnalité, ces PSS se doivent également de présenter un très fort degré d'intégration, c'est-à-dire une forte intensité de l'intervention du confectionneur de bouquet dans la production des effets utiles pour le client (Moati, 2008 : 32-33). Une progression dans leur degré d'aboutissement est clairement visible dans ce qu'Oksana Mont (2004 : 20) identifie comme la plus répandue des typologies de PSS, qui en dénombre trois catégories :

- PSS orientés produit (product-oriented PSS) : fournit un service additionnel au produit vendu ;

- PSS orientés usage (use-oriented PSS) : l'usage du produit est vendu, non le produit lui-même ;

- PSS orientés résultat (result-oriented PSS) : le producteur garantit la satisfaction des besoins du consommateur, quelle que soit la combinaison de produits et services utilisée.

21 La première catégorie regroupe une grande partie des exemples recensés dans la littérature sur les PSS. Consistant à accoler un ou plusieurs services accessoires à un produit (financement, assurance, maintenance, reprise en fin de vie, formation...), ces offres de solutions disposent bien d'un degré de complétude plus élevé que la vente du produit seul. Par contre, le mode de transaction restant inchangé (transfert de la propriété du produit au consommateur), cette catégorie de PSS n'implique ni un élargissement du domaine de responsabilité du producteur, ni une amélioration de leur performance. Dotés ainsi d'un degré d'intégration faible, voire nul, les PSS «orientés produit » ne relèvent donc pas de l'économie de fonctionnalité. De fait, ses préceptes ne transparaissent que dans les cas les plus aboutis de PSS, ceux qui sont "orientés usage » et « orientés résultat ». Leur degré d'intégration est en effet bien plus élevé, puisque les fournisseurs de produit/service y conservent la propriété des actifs matériels supports des offres de solutions, et, dans le dernier cas, prennent eux-mêmes en charge l'ensemble des dépenses liées à leur mise en œuvre. Autrement dit, la responsabilité des producteurs ne se trouve plus ici étendue uniquement à la fin de vie de leurs produits, mais à l'ensemble de leur cycle de vie, y compris et notamment en phase d'utilisation, dont ils ont tout intérêt à optimiser la performance.

Cette typologie de PSS nous permet néanmoins d'identifier d'emblée deux grandes catégories de modèles de revenu compatibles avec la logique de la fonctionnalité : les ventes d'usage et la contractualisation à la performance. Cependant, cette classification ne permet pas de faire ressortir un troisième type de modèle ${ }^{6}$ qu'on trouve référencé plus explicitement dans d'autres typologies. Il s'agit des «services d'usage partagé » (shared utilisation services) (Roy, 2000), aussi connus sous le nom de « consommation collective » ( collective consumption) (Cooper et Evans, 2000), autrement dit des modèles économiques 
qui font appel à un mode de transfert entre individus bien particulier : la mutualisation de biens et de services.

Nous nous proposons donc de référencer les applications de l'économie de fonctionnalité en fonction de trois catégories de modèles de revenu :

- la consommation collective: location de courte durée, auto-partage, laveries automatiques... ;

- les ventes de fonctions d'usage : leasing, ventes fonctionnelles ;

- la contractualisation au résultat : contractualisation à la performance énergétique, facilities management, approvisionnement à moindre coût, protection biologique intégrée.

Ce sont ces différents modèles économiques d'entreprise que nous allons entreprendre de répertorier dans la suite de cette partie, essentiellement sous l'angle de leur modèle de revenu. Après une description succincte de leur mécanisme financier, des exemples d'initiatives permettront d'illustrer les types d'offres qu'il est possible de leur associer, sans pour autant prétendre constituer une présentation complète de leur proposition de valeur. Nous nous efforcerons enfin pour chacun de montrer en quoi il ouvre la possibilité de réduire la pression environnementale associée à l'acte de production et de consommation, par rapport à la vente « classique » de produits.

\subsection{La consommation collective}

La consommation collective regroupe les offres de biens et services qui sont utilisés successivement par plusieurs clients. Bien qu'elles soient en général rangées dans la catégorie des offres de solutions "orientées usage »- ce qu'elles sont en effet - nous avons choisi de leur consacrer une catégorie à part entière en raison du fait que les gains environnementaux qu'elles permettent de réaliser découlent en grande partie d'une intensification de l'usage des produits, ce qui n'est pas le cas des offres qui sont destinées à des clients uniques.

\subsubsection{La location de courte durée}

La location est un modèle transactionnel dans lequel le client obtient contre paiement la possession temporaire d'un produit, sur une durée allant typiquement de quelques heures à quelques jours. Le dépôt d'une garantie (dont le montant varie généralement entre $0 \%$ et $15 \%$ du prix du bien) est généralement demandé lors de l'établissement du contrat de location, afin de prévenir le loueur contre les risques de défaut de paiement ou de détérioration du bien par le locataire.

La location ponctuelle ou de courte durée de biens est une pratique bien connue en France, nous ne nous attarderons donc guère dessus. Citons juste une référence désormais incontournable, à savoir le site internet Zilok, qui propose depuis 2007 une plate-forme de location entre particuliers, système de contractualisation et d'assurance compris. Fort de son succès, Zilok a ouvert son site aux professionnels de la location, qui peuvent déposer leur catalogue de produits en ligne moyennant le paiement d'un abonnement. En 2011, Zilok annonçait détenir un répertoire de 200000 produits disponibles en location à travers la France et gérer 6000 demandes par mois.

L'intérêt environnemental principal de la location provient du fait qu'il est fait usage des fonctionnalités du produit de manière plus intensive que s'il était possédé par un individu unique qui ne s'en servirait sinon souvent qu'occasionnellement, ce qui permet de 
maximiser la valeur d'usage des produits et d'en réduire le nombre nécessaire à la satisfaction des besoins des individus. La possibilité de pouvoir louer conduit ainsi souvent à la décision de ne pas acheter, et donc produire, des biens spécifiques qui sont assez onéreux compte tenu de leurs fréquences et durées d'utilisation. Mais la location peut aussi avoir des effets pervers, puisqu'elle permet d'accéder temporairement à la possession de produits auxquels un individu isolé n'aurait jamais pu accéder. Or, si le produit est gourmand en énergie lors de son utilisation, le fait que son usage se démocratise n'est pas forcément souhaitable, puisqu'il peut conduire à un effet rebond. En outre, en lui permettant d'accéder ainsi à des technologies jusque-là hors de son atteinte, le rythme auquel les nouveaux produits sont perçus comme des nécessités est accru, ce qui contribue à en promouvoir la consommation. Qui plus est, il est connu que les produits de location sont susceptibles d'être soumis à des comportements non précautionneux de la part des usagers, pouvant conduire à une dégradation prématurée des produits.

\subsubsection{L'autopartage}

L'autopartage (en anglais car sharing) est un système dans lequel un ou plusieurs véhicules sont mis à la disposition d'un groupe d'utilisateurs pour le trajet de leur choix et pour une durée limitée. Dans ce modèle, qui est très proche dans son principe de la location, une société ou une agence publique met à disposition des abonnés du service une flotte de véhicules, selon le principe de l'accès en libre-service: les utilisateurs réservent le véhicule de leur choix par internet ou par téléphone, pour des durées généralement courtes, en prennent possession de manière automatique (à l'aide d'une carte d'accès électronique) dans la station qu'ils ont définie, et le restituent, toujours de manière automatique, à n'importe quelle heure du jour ou de la nuit, soit dans la station initiale, soit dans l'une des autres stations du réseau. Le service leur est facturé périodiquement, selon des grilles tarifaires qui varient suivant les opérateurs : au forfait, au temps d'utilisation et/ou au kilométrage (avec ou sans frais d'abonnement et frais de prise en charge fixes). Quel que soit le mode de facturation retenu, ces tarifs comprennent l'ensemble des coûts liés au service (carburant, assurances, entretien, amortissement du véhicule, impôts et taxes divers).

L'exemple d'autopartage le plus connu en Europe est probablement celui de la société suisse Mobility Carsharing ${ }^{7}$, qui comptait 96800 clients en 2010 et disposait d'une flotte de 2500 véhicules, répartis sur un réseau de 1250 emplacements. Bien que plusieurs dizaines de villes françaises proposent leurs propres services d'autopartage, le marché français reste encore à l'heure actuelle bien moins développé qu'il ne l'est en Suisse. Il ne suffirait cependant que d'un coup de pouce des pouvoirs publics pour encourager le développement de ce type d'intiatives et que ce retard soit rapidement comblé, en répliquant par exemple l'initiative de la Mairie de Paris. Forte du succès de Vélib' (système de location en libre-service de vélos), celle-ci vient en effet de mettre en place Autolib', un service d'autopartage qui met à disposition des habitants de Paris et sa proche couronne 3000 automobiles électriques disponibles dans plus de 1000 stations (Syndicat Mixte Autolib', 2010 $)$. Et manifestement, le marché de l'autopartage semble être particulièrement porteur, puisqu'il connait actuellement une progression foudroyante aux États-Unis, alors qu'il y était encore quasiment inconnu il y a à peine une dizaine d'années. L'entreprise Zipcar', notamment, qui compte actuellement 693000 membres pour une flotte de 8900 véhicules répartis sur une vingtaine de grandes villes 
aux États-Unis, au Canada et en Grande-Bretagne, et sur plusieurs centaines de campus universitaires, connait actuellement une croissance continue de l'ordre de $30 \%$ par an, et semble bien disposée à asseoir sa domination sur ce marché en plein boom, comme le montre sa politique d'expansion continue et de rachat de ses concurrents.

Les avantages environnementaux de l'autopartage sont essentiellement les mêmes que ceux de la location. L'autopartage permet ainsi de maximiser l'usage des véhicules et de réduire le nombre de véhicules en circulation de manière significative : chaque véhicule auto-partagé permettrait ainsi de retirer quinze véhicules personnels de la circulation (Millard-Ball, 2005 : 4-11). La pratique de l'autopartage a également une influence sur les comportements et les choix des utilisateurs en matière de mobilité. Une étude réalisée en 2010 par le groupe Frost \& Sullivan estime que les utilisateurs de l'autopartage parcourent des distances $31 \%$ moindre que celles qu'ils couvraient quand ils possédaient un véhicule personnel (Zhao, 2010), et une étude menée par l'office Fédéral de l'Énergie de la ville de Berne pour le compte de Mobility montre une tendance de ces usagers à se débarrasser de leurs voitures et à favoriser l'intermodalité des transports, en privilégiant notamment l'usage des transports en commun. Du fait des changements de modalités de transport de ses clients, d'une part, et que sa flotte de véhicules est $26 \%$ plus économe que la flotte des voitures privées suisses, d'autre part, cette même étude estime que la société Mobility permet de réduire les émissions de $\mathrm{CO}_{2}$ de ses usagers d'environ $290 \mathrm{~kg}$ par an, par rapport à une situation sans autopartage (Haefeli et al., 2006 : XII).

Notons que la consommation collective ne se limite pas à la location de courte durée et à l'autopartage. Les laveries automatiques, bien connues en France, ou le partage de l'accès à une machine à laver entre habitants d'un immeuble, comme il se fait en Suisse et en Allemagne $^{10}$, en sont d'autres exemples.

\subsection{Les ventes de fonctions d'usage}

La catégorie des ventes de fonction d'usage regroupe les offres de solutions consistant à mettre à disposition d'un client unique, pour une durée relativement longue, l'usage d'un produit dont le prestataire de l'offre conserve la propriété. Les gains environnementaux que ces modèles économiques peuvent permettre de réaliser sont proportionnés aux mesures que ce dernier met en œuvre afin de maximiser la valeur économique qu'il tire de ses actifs.

\subsubsection{Le leasing}

Le leasing est une technique contractuelle par laquelle une société fabrique ou achète un bien puis le loue à un individu ou une entreprise pour une durée correspondant généralement à sa durée de vie économique, c'est-à-dire celle de son amortissement fiscal. Le crédit-bailleur reste propriétaire du bien pendant la durée du contrat et ne fait qu'en fournir la possession à son cocontractant. Selon le type de contrat de leasing qu'il a signé, le crédit-preneur peut avoir plusieurs choix à l'issue du bail. Il peut tout d'abord décider de lever l'option, c'est-à-dire d'acquérir le bien contre le versement d'une somme correspondant à sa valeur résiduelle, qui est généralement assez faible en regard de sa valeur initiale. Ce cas de figure, qui revient de fait à pratiquer une location-vente (capital leasing), n'est pas du domaine de l'économie de fonctionnalité. Il ne s'agit en effet que d'une sous-traitance d'achat, aboutissant au transfert définitif de la propriété d'un bien 
au moyen d'une manipulation financière fortement similaire à un crédit bancaire. Le cas de figure qui nous intéresse bien davantage est celui du crédit-bail (operational leasing) aussi appelé en France, pour les particuliers, location avec option d'achat - dans lequel le crédit-preneur choisit en fin de contrat de restituer le bien au bailleur ou de reconduire la location pour une nouvelle durée, en général avec du matériel neuf. C'est bien entendu parce que le produit reste la propriété du crédit-bailleur sur l'ensemble de son cycle de vie que nous voyons en ce type de transaction un modèle économique compatible avec les principes de l'économie de fonctionnalité.

Le leasing de moquette, dans lequel s'est lancée au cours des années 1990 l'entreprise Interface Inc., fut longtemps présenté comme un échec retentissant. C'est là une erreur que nous allons entreprendre de corriger. Reconnaissons pour commencer qu'il est vrai qu'en 2000, soit six ans après le lancement du programme "Evergreen Lease" d'Interface, celui-ci n'était pas suffisamment rentable pour pouvoir être considéré comme un succès, d'un côté à cause du faible coût des matières premières, ce qui rendait le recyclage de nylon plus coûteux que la fabrication de moquette à partir de matériaux vierges, et d'un autre côté en raison du surcoût de la récupération des moquettes par rapport à leur mise en décharge, que les clients ne se montraient pas disposés à payer - d'où un faible taux de retour des produits usagés (Fishbein et al., 2000: 40). Le bilan n'était cependant pas totalement négatif pour Interface, puisque le groupe avait tout de même réussi à réduire ses besoins en nylon dans la production de ses moquettes de plus d'un millier de tonnes grâce à ses diverses innovations en termes de matériau et de conception de ses produits et à économiser tout de même 145 millions de dollars entre 1994 et 2000 grâce à ses efforts d'élimination des pertes et déchets dans ses produits et ses procédés (Fishbein $e t$ al., 2000 : 39). Probablement eut-il été plus opportun de parler à l'époque de bilan mitigé, voire de succès d'un point de vue environnemental, et de soulever l'hypothèse qu'Interface était peut-être trop en avance sur son temps ? Un autre détail aurait déjà dû instiller le doute quant à la validité de ce constat d'échec: bien que leurs initiatives se soient aussi soldées par des déboires économiques, plusieurs des concurrents d'Interface sur les marchés du nylon et de la moquette (Collins \& Aikman, DuPont, BASF, Milliken) s'étaient eux aussi lancés à l'époque dans des initiatives visant à récupérer des moquettes en fin de vie à des fins de recyclage (Fishbein et al., 2000 : 33). Malgré des soucis évidents de rentabilité, la compétition était déjà forte sur le marché américain de la récupération des moquettes usagées. Dix ans plus tard, InterfaceFLOR annonçait le lancement d'investissements importants dans l'amélioration de son processus ReEntry (la partie recyclage du programme) sur son lieu de production de Scherpenzeel (aux Pays-Bas), et son intention d'étendre le concept Evergreen Lease à la moquette modulaire ${ }^{11}$. De son côté, DuPont s'était lancé dans le même temps dans le développement d'un système de franchise dans lequel il convertit les vendeurs de moquettes existants à son "DuPont Flooring System", dans le but d'accélérer la pénétration sur le marché de son modèle déposé de fibre de tapis en nylon (Seid, 2011). C'est qu'en dix ans, beaucoup de choses ont changé. La réglementation a évolué et la mise en décharge de tapis et moquettes usagés est désormais facturée au consommateur américain, coût dont il peut s'exonérer en justifiant de la récupération et du recyclage de ces produits en fin de vie par l'une des sociétés habilitées. À ce facteur incitatif, qui a permis de lever la barrière du manque d'implication des utilisateurs finaux, vient s'ajouter un élément conjoncturel sur lequel les analystes financiers de l'industrie du nylon avaient semble-t-il parié à juste titre : la hausse du prix du pétrole, dont le nylon est un dérivé direct. Alors qu'il était en 2000 à un 
de ses niveaux le plus bas, le prix de cette matière n'a depuis cessé d'augmenter, rendant le leasing et le recyclage de tapis et moquettes chaque année plus rentable. En 2007, Interface annonçait ainsi avoir doublé ses revenus, quasiment doublé le nombre de ses employés et triplé ses profits grâce au passage à un tel modèle économique. En 2009, soit en pleine crise financière, la valeur boursière de l'entreprise affichait une augmentation de $550 \%$ sur cinq ans (Scott, 2009: 143-144). D'un point de vue environnemental, les chiffres sont tout aussi impressionnants : les dalles de moquettes produites comprennent entre $39 \%$ et $71 \%$ de matériaux recyclés, et pour chaque $\mathrm{m}^{2}$ de moquette, le volume de déchets envoyés en décharge été réduit de $88 \%$ et la consommation globale d'énergie réduite de $47 \%$ entre 1996 et $2011^{12}$.

À l'exception notable des véhicules automobiles, le leasing est une offre qui reste essentiellement destinée aux entreprises. La société Lokéo est l'une des premières à avoir osé s'attaquer au marché français du leasing aux particuliers, en lançant en juin 2010 différentes formules de location de longue durée (avec reprise en fin de contrat, autrement dit de leasing "sans option d'achat») de produits électroménagers et multimédia. La principale limite sur le plan environnemental de l'offre de Lokéo est que cette société ne joue qu'un rôle d'intermédiaire entre le producteur et le client, et n'a donc guère d'influence sur la façon dont les produits sont conçus. Elle semble néanmoins avoir mis en place une politique proactive en matière de gestion des produits en fin de contrat, puisque ceux-ci sont, selon les cas et par ordre de priorité, nettoyés, reconditionnés et remis en location, revendus sur le marché d'occasion, utilisés dans le cadre de parcs de prêt ou envoyés dans un circuit de recyclage des $\mathrm{DEEE}^{13}$. Un an après le lancement de son activité, son succès était déjà tel que l'entreprise avait décidé d'étendre la gamme de ses produits (caves à vin, hottes, fours et cuisinières encastrables...) et de ses services (installation, initiation à la prise en main et paramétrage des ordinateurs...). Elle envisageait par ailleurs de lancer une offre "pro", suite à la demande de nombreuses PME souhaitant s'équiper en télévisions et ordinateurs, et de partir à la conquête du marché européen, en commençant par la Belgique ${ }^{14}$.

Les avantages environnementaux du leasing résident dans le fait qu'il est dans l'intérêt du producteur de mettre en place des programmes de réutilisation et recyclage de leurs produits, et d'une manière générale de boucler les flux de matières et d'énergie de manière à optimiser la valeur résiduelle de ses produits. Il s'agit cependant d'une démarche qui est loin d'être systématique. Toutes les formes de leasing ne conduisent en effet pas à un retour du produit au producteur à la fin de sa période d'exploitation, et même dans le cadre d'un crédit-bail, le produit en retour peut être revendu par le producteur sur le marché de l'occasion voire, si c'est une société intermédiaire qui gère les contrats, ne jamais lui revenir. De plus, l'engagement du producteur dans des programmes de l'ampleur de ceux d'Interface dépend de plusieurs facteurs: les caractéristiques du produit, dont la valeur résiduelle peut être insuffisante (ou les coûts de démantèlement et d'enfouissement insuffisamment élevés) en regard des coûts supplémentaires générés par une politique de formation du personnel, de maintenance, entretien et remplacement éventuel du matériel défectueux, et surtout de récupération et rénovation/recyclage des produits usagés; sa volonté ou sa capacité à engager les réformes nécessaires en matière d'organisation et de conception; tout simplement sa compréhension de l'intérêt que présentent de tels modèles économiques pour lui, et pour l'environnement, par rapport à la vente de produits dont il n'a plus à assumer la responsabilité à partir du moment qu'ils ne lui appartiennent plus. 


\subsubsection{Les ventes fonctionnelles}

38 le produit per se (on peut y voir en ce sens l'application la plus «pure » des principes de l'économie de fonctionnalité). Typiquement, au lieu d'acheter un photocopieur, le client paie à la copie et donc en fonction de l'usage du bien à sa disposition. Bien que les ventes fonctionnelles prennent souvent la forme d'un crédit-bail dans les relations BtoB (Business to Business, autrement dit entre entreprises), ainsi que le note Oksana Mont (2004:17-18), elles s'en distinguent dans la pratique sur plusieurs points. Au lieu de simplement louer un produit, le fournisseur y met en effet en œuvre une combinaison de produits, consommables et services afin que son client puisse bénéficier de la fonction délivrée par le produit sans avoir à en être propriétaire, à l'entretenir, le réparer ou s'en débarrasser en fin de vie. Le client payant à l'usage, les coûts additionnels qu'il encoure dans une location ou un crédit-bail (consommables, entretien...) deviennent ici transparents pour lui. Au lieu de rembourser un emprunt ou de payer un loyer, le service devient pour lui une dépense courante dont le montant ne dépend que du niveau de ses besoins, et qui cesse à la disparition de ces derniers. Le fournisseur est de son côté libre d'utiliser une combinaison de produits neufs ou de seconde main pour délivrer la fonction sur laquelle les deux parties se sont entendues.

La compagnie Electrolux s'est intéressée assez tôt à ce modèle économique. Son premier essai dans le domaine remonte à 1995, quand sa filiale Euroclean s'est lancée avec succès dans la vente de la fonction de son "scrubber dryer" à la plus grande compagnie de nettoyage industriel de Suède, pour être utilisé dans le cadre de l'entretien des sols d'un grand supermarché de Stockholm. Le programme de ventes fonctionnelles de la compagnie Euroclean, qui développa également d'autres contrats, prit fin en 1998, quand Electrolux la revendit à un autre conglomérat industriel. Cependant, depuis le test d'Euroclean, le concept de vente fonctionnelle est devenu partie intégrante de la stratégie d'Electrolux, qui continua depuis à essayer de garder un contact plus proche avec ses produits tout au long de leur cycle de vie à l'aide de contrats de services, d'extensions de garanties et de crédit-bails, bien que ceux-ci portent sur des produits spécifiques au lieu d'une fonction « sans support fixe » (Fishbein et al., 2000 : 42-43). Décidant de s'attaquer au marché des particuliers, Electrolux s'est lancé en 1999 dans une nouvelle expérimentation sur l'île de Gotland, en Suède. L'idée du projet "Pay-per-Wash" était de vendre aux consommateurs une fonction de nettoyage de vêtements en les faisant payer par cycle de lavage accompli. Chacun des 50 foyers recrutés sur l'île reçut ainsi une machine à laver dotée d'un compteur électronique permettant d'effectuer le suivi des cycles d'utilisation de la machine, qu'Electrolux s'était engagé à remplacer après mille cycles de lavage. Le fait de facturer aux clients une faible somme lors de l'installation, puis de les faire payer à chaque lavage était censé les encourager à n'utiliser la machine qu'à charge pleine, et donc de réduire les volumes d'eau, de détergent et d'énergie consommés ${ }^{15}$. Les machines en retour étaient pour leur part destinées à être rénovées ou recyclées. Il est difficile de savoir si ce programme a eu le temps d'avoir une incidence sur le comportement des cobayes, l'expérience ayant été arrêtée au bout d'un an sur les quatre prévus. La raison en est la perte de contrôle du service par Electrolux, qui était devenue totalement dépendante de la société qui contrôlait les compteurs électroniques et s'occupait de la facturation du service. Celle-ci aurait ainsi pu utiliser des compteurs de

Développement durable et territoires, Vol. 5, $n^{\circ} 1$ | Février 2014 
moindre qualité ou augmenter le prix de ses services sans qu'Electrolux ait le moindre recours en la matière (Stahel, 2003).

De même que le leasing tel que pratiqué par Interface, les ventes fonctionnelles sont porteuses d'incitations fortes à réaliser des économies, tant sur le plan financier qu'environnemental. Puisque le prestataire peut s'accorder la possibilité de fournir à ses clients des machines de seconde main, du moment que celles-ci assurent la fonction attendue, il est incité à réduire sa production de machines neuves et d'extraire la valeur résiduelle des machines en fin de vie en les rénovant et les réutilisant, ou en recyclant les éléments hors d'usage. De plus, puisqu'il a également à sa charge l'approvisionnement des supports fonctionnels en consommables, il est dans son intérêt de fournir une fonction qui en utilise le moins possible, par exemple en formant ses clients à une utilisation de ses machines minimisant les consommations de ressources et l'usure des machines. On peut tirer de l'échec relatif de la seconde expérience d'Electrolux un enseignement important en matière de réussite, ou non, des offres reposant sur la vente de fonctionnalités : leur succès dépend non seulement de la maîtrise de l'ensemble du cycle de vie du produit (ne serait-ce que par l'intermédiaire de solides contrats de soustraitance), mais aussi de l'ensemble de la chaîne de distribution du service, au risque de se voir court-circuité par un de ses partenaires avals, plus proche du client.

\subsection{La contractualisation au résultat}

41 La contractualisation au résultat est comme son nom l'indique un modèle économique dans lequel un prestataire s'engage contractuellement auprès d'un client sur l'obtention de résultats, plutôt que sur la mise en place de moyens, dans le cadre d'une offre de solution portant sur la prise en charge de l'ensemble des actions nécessaires à l'obtention d'une fonction. Les risques et les coûts étant à la charge du prestataire, il est dans l'intérêt de celui-ci de minimiser les flux de matières et d'énergie.

\subsubsection{La contractualisation à la performance énergétique}

La contractualisation à la performance énergétique n'est pas un modèle transactionnel récent, puisqu'il fut utilisé au début de la révolution industrielle par James Watt, inventeur de la machine à vapeur à chambre de condensation séparée, et son associé Matthew Bolton pour convaincre leurs clients d'investir dans une technologie jusqu'alors inconnue, et assez coûteuse. Ils utilisèrent pour cela une tactique commerciale assez inventive : plutôt qu'un crédit-bail ou un crédit à taux variable, ils leur proposèrent de ne rien leur reverser de plus qu'une partie des économies réalisées grâce à leurs engins, calculées sur la base du coût du fourrage auparavant utilisé pour nourrir les chevaux qui faisaient le travail de leurs «chevaux-vapeurs» (Zaring et al., 2001 : 267). C'est sur le même type d'argumentation marketing et sur des termes similaires que s'appuient de nos jours les contrats de performance énergétique ${ }^{16}$.

Les Sociétés de Service en Efficacité Énergétique (SSEE), aussi souvent connus depuis plusieurs dizaines d'années dans de nombreux pays sous le nom d'Energy Services Companies (ESCos), s'engagent auprès de leurs clients au travers de contrats de performance portant sur des objectifs de qualité de service et d'amélioration sur la durée de l'efficacité énergétique de leurs installations. Ces objectifs sont définis sur la base de critères chiffrés et mesurables (température, disponibilité, économies, niveaux de 
rejets...) et suivis au moyen d'indicateurs de performances. Outre la fourniture d'une utilisation finale de l'énergie qui soit efficace (par exemple l'approvisionnement en chaleur, éclairage, réfrigération, production d'eau chaude ou de vapeur...), les contrats de performance engagent les SSEE à réaliser tout un ensemble d'actions, comprenant entre autres la réalisation d'un diagnostic initial, la définition de préconisations en vue de l'amélioration de l'efficacité énergétique, le financement des investissements, la planification et l'exécution des travaux (par exemple d'isolation thermique du bâti) et de l'installation des équipements requis, l'exploitation et la maintenance des installations, le suivi de leur bon fonctionnement pendant toute la durée du contrat, etc. La mise en œuvre des contrats de performance des SSEE suppose ainsi le déploiement de tout un ensemble de prestations complémentaires, allant du conseil et partenariat dans les domaines techniques et financiers jusqu'à l'installation et la conduite optimale des installations, en passant par la maîtrise des mécanismes d'achat d'énergie ou encore la gestion de dispositifs réglementaires comme les permis d'émissions ou les certificats d'économies d'énergie.

La ville de Berlin (Allemagne) offre un bon exemple de l'efficacité de la contractualisation à la performance énergétique, appliquée ici au secteur public. Dotée d'un budget serré qui ne lui permettait pas de rénover et moderniser ses infrastructures publiques, elle fit appel en 1996 à l'Agence de l'énergie de la ville de Berlin, qui mit en place un programme de partenariat avec plusieurs ESCos en vue de réaliser des économies d'énergie dans plus de 1300 bâtiments publics (dont la mairie de la ville, plusieurs écoles et universités, les bains publics, différents immeubles administratifs...). Les investissements réalisés par les ESCos furent financés grâce aux économies réalisées, permettant à la ville de réduire ses émissions de $\mathrm{CO}_{2}$ de 60000 tonnes et de réaliser des économies financières de l'ordre de dix millions d'euros, sans avoir à aucun moment à débourser le moindre Euro (Fandel et Zuleeg, 2008: 32). Le taux de réduction des consommations énergétiques sur l'ensemble des sites concernés est de $26 \%$ en moyenne ${ }^{17}$.

Les aspects centraux des contrats de performance énergétique sont l'engagement de la SSEE sur une garantie de résultats pendant toute la durée du contrat, et un mécanisme de financement consistant à rembourser les investissements initiaux et à rémunérer la prestation sur la base des économies réalisées par rapport à la situation de départ. Ceci implique que la SSEE assume la plupart des risques - en dehors du risque de baisse du prix de l'énergie, dont le client peut assez aisément convenir qu'il est assez faible - et des coûts liés au projet. Dans le cas où le résultat égale ou dépasse les objectifs fixés, la SSEE et son client vont se partager les économies réalisées, selon le barème défini par le contrat (dont il existe différents types, les plus répandus étant "guaranteed savings" et " shared savings"). Si le résultat est au contraire inférieur aux objectifs fixés, la SSEE doit fournir à ses frais le supplément d'énergie nécessaire à l'obtention du service prédéfini ou, si l'approvisionnement d'énergie n'est pas compris dans le contrat, payer les sommes correspondant au dépassement de consommation. Le modèle d'affaires des SSEE est donc fondamentalement différent de celui des énergéticiens conventionnels, dont l'intérêt est de maximiser les ventes d'énergie, et des cabinets d'ingénierie, qui sont payés pour leurs conseils et ne prennent aucun risque quant aux résultats de leurs recommandations. C'est au contraire grâce à la maximisation des économies d'énergie qu'elles se sont engagées à faire faire à leurs clients que les SSEE vont pouvoir augmenter leurs profits. On retrouve bien ici les mêmes implications économiques - et environnementales - que celles qui découlent des principes théoriques de l'économie de fonctionnalité. 


\subsubsection{Le Facilities Management} d'une demande croissante de la part d'entreprises souhaitant se centrer sur leur cœur de métier et, dans un souci de simplicité, externaliser des processus de plus en plus contraignants (multiplicité des acteurs impliqués, technicité de plus en plus pointue des installations, coûts des espaces de travail de plus en plus élevés...) auprès d'un interlocuteur unique. Il regroupe des prestations classiquement divisées en deux catégories :

- la gestion multitechnique, qui comprend la gestion des énergies et des fluides (électricité, gaz, eau, air comprimé, vapeur...), la maintenance des installations (climatisation, ascenseurs, portes automatiques, réseaux de télécommunications...) et la gestion immobilière (contrats de location, conseil juridique et fiscal, comptabilité...) gardiennage, sécurité, nettoyage, entretien des espaces verts, enlèvement des déchets... et les services généraux (accueil, standard, courrier, reprographie, gestion pour compte de tiers, restauration collective, gestion de la flotte automobile, gestion des espaces de travail, déménagements...). un engagement sur des résultats, le Facilities Management peut être considéré comme une extension des contrats de performance à des gammes de prestations plus globales, ici à destination du parc immobilier non résidentiel.

Notons la proximité de tels modèles d'affaires avec le modèle virtuel de « Home Services Companies " (HSCos) proposé par Tim Cooper et Sian Evans (2000). De telles compagnies seraient en charge de pourvoir un logement et la plupart des formes de services qui y sont associés à des particuliers, ceux-ci payant une rente mensuelle constituée d'une part variable - à tarif compétitif garanti - pour l'énergie, l'eau et les communications, et d'une part fixe pour le bâtiment, son entretien et des services généraux tels que la sécurité, le blanchissage et le nettoyage. Les immeubles d'appartements ou les lotissements pourraient également se voir dotés de centres de compostage et de recyclage, de centrales de cogénération ou autres sources d'énergies renouvelables, et avoir accès à des ressources mises en commun, comme des outils, du matériel informatique ou des équipements sportifs. Pour impliquer les résidents dans les programmes de réduction des flux, des compteurs intelligents pourraient être installés, qui afficheraient en temps réel les consommations d'énergie et d'eau, l'évolution du montant prévu des factures, et des informations sur les moyens de réduire les coûts au maximum tout en maintenant un niveau de confort optimal. « De telles compagnies seraient le plus probablement développées par des promoteurs immobiliers travaillant en partenariat avec des sociétés d'investissement et de crédit immobilier, des propriétaires fonciers, des entreprises de la construction, des énergéticiens ou des locataires" (Cooper et Evans, $2000: 27)$, une des questions restant en suspens, de l'avis des auteurs, étant celle de la légalité d'un contrat qui lierait ainsi les clients à un fournisseur unique.

\subsubsection{L'approvisionnement à moindre coût}

51 L'approvisionnement à moindre coût (ou least cost supply), est une stratégie économique consistant à minimiser les coûts de façon à maximiser les profits. Certains fabricants de 
produits chimiques et phytosanitaires la mettent en œuvre au travers de chemical management services, services de prise en charge intégral de la gestion des produits chimiques de leurs clients incluant la formation du personnel, le respect de la réglementation, le traitement des déchets, etc. Plutôt qu'être vendus au prorata des volumes consommés, les produits chimiques sont vendus dans le cadre d'offres de leasing, de ventes fonctionnelles (c'est-à-dire sur la base du nombre de pièces qu'ils permettent de traiter) ou de contrats de performance (similaires aux CPE) dans lesquels le client n'achète plus un produit mais un résultat, et où le fournisseur se rémunère sur une base comprenant une part fixe et une part variable, cette dernière correspondant à une fraction des économies qu'ils permettent à leurs clients de réaliser.

La société Safechem s'est montrée particulièrement proactive dans ce domaine en développant très tôt des conteneurs - les "Safetainers ", depuis renommés "système Safecare »- qui permettent de récupérer les solvants chlorés en évitant les pertes par évaporation, et en offrant à ses clients un programme de leasing de solvants reposant sur un principe de bouclage des flux : récupération des solvants usagés de ses clients, qui leur sont renvoyés après recyclage. Safechem est désormais passée à l'étape supérieure en développant le programme "Complease ", dans lequel elle offre une solution complète de nettoyage d'outils et de pièces métalliques ajoutant aux services déjà énumérés l'installation d'une machine, sa maintenance, la formation du personnel et des services de suivi de performance et de contrôle incluant l'analyse et la traçabilité des flux de solvants et de déchets ${ }^{18}$. Plusieurs autres sociétés initialement spécialisées dans la vente de produits chimiques se sont également lancées dans des offres similaires, comme Ashland Inc., Chrysler Corp., Ciba AG Division pigment, ou encore Castrol Industrial North America, Inc., dont le programme "Castrol+Plus » a par exemple permis de réduire de plus de $50 \%$ les volumes de liquides de refroidissement consommés - et les déchets de plus de $90 \%$ - dans une usine de Navistar International (White et al., 1999 : 68).

La protection biologique intégrée (Integrated Pest Management) est une autre forme d'approvisionnement à moindre coût, appliquée ici aux pesticides. Comme les autres offres de service "orientées résultat", elle comporte un ensemble de procédures d'évaluation et de contrôle destinées à s'assurer que les objectifs fixés - faire des économies en réduisant les quantités de produits consommés - ont bien été atteints. Il existe cependant une différence en ce qui concerne les moyens qui sont mis en œuvre pour arriver à ces fins, puisqu'il ne s'agit pas de se focaliser uniquement sur la réduction de flux pré-existants, mais autant que possible de substituer à l'utilisation de pesticides une combinaison de pratiques "issues du bon sens». La première étape est ainsi le passage en revue des espèces présentes sur site et la détermination du seuil d'action à partir duquel des mesures devront être mises en œuvre. De nombreux organismes (plantes, insectes...) ne représentent en effet aucun danger pour les cultures, et peuvent même être bénéfiques. La première ligne d'action recommandée est de nature préventive : rotation des cultures et sélection de variétés résistantes. Si des mesures supplémentaires s'avèrent nécessaires, l'étape suivante passe par l'emploi de méthodes «mécaniques» (désherbage et piégeage) ou d'agents naturels, telles que des phéromones destinées à repousser ou perturber la reproduction des nuisibles, ou l'introduction d'espèces prédatrices des nuisibles que l'on cherche à éliminer. L'emploi de pesticides est un dernier recours, les pesticides provenant de sources naturelles étant alors préférés à ceux de synthèse. 
L'entreprise néerlandaise Koppert est le leader mondial de la protection biologique des cultures et de la pollinisation naturelle. Son système "Bio-Plus » est une solution de gestion intégrale des nuisibles à destination des cultures en serre, comprenant, outre le conseil et la formation des clients, un suivi strict de la condition des plantations, effectuée par les experts de Koppert ou directement par les agriculteurs, grâce à un logiciel dédié. Les agriculteurs sont facturés à l'hectare et ne paient pas de charges supplémentaires pour l'utilisation de prédateurs naturels (Goedkoop et al., 1999: 87). L'exemple de Ciba-Geigy est très instructif. Accusée d'empoisonner les riziculteurs de Madagascar avec ses pesticides, et déterminée à démontrer que la charge de la faute était à imputer à ceux-ci et non au produit, cette société a offert un service garantissant l'élimination des nuisibles et un meilleur rendement des rizières, pour un tarif équivalent à ce que lui rapportait ses ventes de pesticides lors de la période incriminée. En décidant quand, où et dans quelles proportions les pesticides devaient être pulvérisés, Ciba-Geigy a réussi en quelques années à en réduire les quantités utilisées de $70 \%$, pour une amélioration de rendement allant jusqu'à $400 \%$. Du fait de l'opposition féroce des anciens distributeurs et revendeurs de pesticides, ce programme a dû être abandonné (Stahel, 2003).

Comme l'ensemble des autres exemples d'application de l'économie de fonctionnalité, l'approvisionnement à moindre coût conduit donc le fournisseur à essayer de minimiser les volumes de produits utilisés de façon à réduire au maximum ses coûts de revient, de fonctionnement, et de traitement des déchets. Le développement de ce type d'offres est notamment encouragé par deux facteurs: d'une part la volonté d'externaliser des fonctions qui ne sont pas centrales à leur cœur de métier et dont la gestion en interne peut s'avérer assez coûteuse - tendance qui va en se généralisant dans tous les secteurs de l'industrie - et d'autre part l'existence de réglementations de plus en plus strictes en matière de gestion des produits chimiques, à l'image du règlement européen REACH. Le pendant négatif de cette stratégie pour les fabricants de produits chimiques est son impact négatif sur leurs ventes directes, que la plupart ne sont guère disposés à abandonner.

\section{L'économie non-marchande de la fonctionnalité}

Les relations contractuelles marchandes ne sont pas les seules formes d'échange qui sont adaptées au modèle économique de la fonctionnalité, et si sa mise en œuvre peut en effet avoir pour but «d'obtenir une meilleure compétitivité et une augmentation des revenus des entreprises " (Stahel, $2006: 145)$, elle peut aussi être déployée au travers de mécanismes qui ne conditionnent pas la satisfaction des besoins des individus à la réalisation d'un profit. Il est assez frappant de constater que la très grande majorité des travaux sur l'économie de fonctionnalité n'envisage celle-ci que dans sa dimension marchande. Or, l'opérationnalisation de l'économie de fonctionnalité ne nécessite pas forcément que la fonction d'usage qui vient en remplacement d'un produit soit vendue. Elle peut aussi faire l'objet d'échanges qui se situent en dehors de la sphère du marché, typiquement de formes de mutualisation de biens et services fondées sur la réciprocité - ce que les anthropologues et les sociologues, dans la lignée de Marcel Mauss, qualifient aussi de don/contre-don (voir par exemple Caillé, 2005). Nous voyons dans cet oubli une preuve de la myopie des sociétés capitalistes qui, aveuglées par la prévalence actuelle du marché, tendent à occulter les modes d'échange non marchands, c'est-à-dire ne s'effectuant pas 
sur la base d'un prix résultant de la rencontre entre l'offre et la demande. Ainsi que le rappelle Karl Polanyi (2008:65-66), ces formes d'intégration comptent pourtant parmi les plus anciennes, alors que le marché autorégulateur n'a pour sa part occupé pendant longtemps qu'une place très marginale dans les échanges entre individus.

C'est dans certaines formes de consommation collective que se perpétue de nos jours l'esprit du don et du contre-don, de l'échange fondé sur la réciprocité plutôt que sur la force ou le marché.

Revenons pour commencer sur l'exemple de l'autopartage, dont les formes commerciales connaissent actuellement un succès foudroyant, en rappelant que nombre de ces entreprises étaient à l'origine des coopératives, et que plusieurs le sont encore. La société Mobility Carsharing est ainsi le fruit de la fusion, en 1997, des coopératives AutoTeilet et ShareCom, qui furent pour leur part fondées en 1987. I-GO Carsharing, à Chicago, est une organisation à but non lucratif américaine qui continue de résister à la stratégie d'expansion hégémonique de Zipcar. Il existe également de nombreuses initiatives d'autopartage coopératif, composées de groupes informels d'individus (voisins, amis...) qui s'associent en vue de l'achat et du partage de l'usage d'un ou plusieurs véhicules. Historiquement le plus ancien, ce mode d'autopartage est aussi le plus répandu en France, bien que son caractère souvent informel et la taille souvent réduite des initiatives rendent difficile toute évaluation précise de son rayonnement. On estime ainsi que l'autopartage dans la sphère privée concernait en 2009 en France entre 35000 et 70000 personnes, et entre 10000 et 20000 véhicules (ADETEC, 2009: 8). Notons que les risques de détérioration des véhicules sont beaucoup plus faibles dans l'autopartage coopératif que dans la location ou l'autopartage commercial, du fait d'une plus grande proximité entre individus. Ceux-ci sont ainsi incités à maintenir le matériel en bon état bien moins par la peur d'une sanction financière que par respect envers les autres membres du groupe - ou du moins, a minima, la volonté de préserver leur réputation...

consommation collective peut également prendre la forme d'une mutualisation avec accès simultané, plutôt que séquentiel, à la fonction d'usage. C'est le cas avec le covoiturage, sur lequel nous ne nous étendrons pas, ou l'achat et l'utilisation en commun de saunas, comme il se pratique dans certains pays nordiques.

Les Systèmes d'Échanges Locaux (SELs) sont une autre forme de consommation collective avec accès (ici généralement séquentiel) à des fonctions d'usage, qui sont cette fois multiples. Il s'agit de réseaux d'échange à but non lucratif dans lesquels les échanges de biens et de services (de la garde d'enfant aux travaux de charpente, en passant par les soins aux personnes ou les cours de musique) sont orchestrés à l'aide d'une monnaie alternative à la monnaie officielle. Souvent purement virtuelles (les échanges sont enregistrés dans une base de données à laquelle ont accès tous les membres du réseau), ces monnaies sont la plupart du temps "sans crédit» (c'est-à-dire que les crédits sont dénués de taux d'intérêt, ce qui empêche la monnaie de "gonfler » au fur et à mesure des transactions) et «fondantes » (elles perdent de la valeur au fil du temps, ce qui incite à utiliser rapidement ses avoirs, plutôt que de thésauriser). Au lieu de s'envoler vers des placements lointains et ne quasiment pas circuler dans l'économie réelle, les monnaies complémentaires tournent du fait de ces caractéristiques beaucoup plus vite que l'Euro, ce qui permet d'engendrer de la richesse à l'échelle locale. Il existait en 2011 « plus de 4000 systèmes à travers le monde, touchant une cinquantaine de pays et environ un million de personnes » (Leblanc, 2011). En mettant l'accent sur la convivialité, la transparence et la participation de tous, ces systèmes sont généralement considérés plus «durables » que 
l'économie de marché, puisqu'ils permettent aux individus de répondre à leurs besoins de manière autonome, de réduire les coûts et la pollution dus aux transports, et de changer les modes de consommation en promouvant la mutualisation, le recyclage et la maintenance. Autant de raisons tendant à confirmer l'intuition de Paul-Marie Boulanger selon laquelle «des systèmes tels que les SEL ont un rôle à jouer dans le développement de cette économie de fonctionnalité, (...) avec cet avantage supplémentaire qu'il s'agirait d'abord, dans toute la mesure du possible, de substituer en priorité le partage, le prêt ou l'échange à la vente du service en question » (Boulanger, $2007: 34$ ).

61 Si l'on accepte de mettre de côté l'idée selon laquelle l'économie de fonctionnalité ne pourrait se concrétiser qu'au travers de "modèles de revenu » d'entreprises, autrement dit de transactions contractuelles axées avant tout sur la recherche du profit, force est de constater qu'on retrouve dans les SELs et dans toutes les formes de consommation collective favorisant le partage et la mutualisation, la même logique : substituer l'accès à une fonction d'usage à la propriété privée d'un bien.

Afin d'élargir la compréhension que l'on peut avoir du concept d'économie de fonctionnalité, nous terminerons cette partie en en proposant une définition dont la formulation - que nous empruntons en grande partie à Dominique Bourg et à Nicolas Buclet - permet de ne pas exclure d'emblée les modes de transaction alternatifs au marché : l'économie de fonctionnalité consiste en la substitution de l'accès à une fonction d'usage à l'appropriation privative d'un bien matériel (ou d'un process).

\section{Conclusion}

L'économie de fonctionnalité est un concept qui rencontre un succès grandissant auprès des responsables d'entreprise et des décideurs politiques, ce qui est assez compréhensible de la part d'un modèle qui, dans un monde de raréfaction et de hausse du prix des ressources, préconise d'engendrer de la croissance économique grâce à la réduction des flux de matières et d'énergie. Force est cependant de constater que, si les bases théoriques de ce modèle économique alternatif à la vente de produits sont en général bien comprises, il n'en est pas toujours de même de ses modalités de mise en œuvre. Certains s'évertuent ainsi à croire reconnaître des applications de l'économie de fonctionnalité dans des offres de solutions qui ne sont en fait que des ventes de produits déguisées. À l'inverse, on tend trop souvent à oublier qu'elle peut aussi se déployer au travers d'échanges non marchands.

Les exemples d'offres de solutions fonctionnelles évoqués dans cet article permettent de mettre en évidence que le passage à un modèle économique reposant sur la vente de fonctions d'usage ou de contrats de performance peut s'avérer très profitable, d'un point de vue économique autant qu'écologique. Ils montrent également que les entreprises qui choisissent d'effectuer un tel basculement peuvent se trouver confrontées à différentes sortes de difficultés. Cela suppose en effet souvent un changement profond de leur mode de fonctionnement qui, au-delà d'une simple refonte de leur schéma organisationnel, nécessite de développer tout un ensemble de nouvelles compétences, d'envisager leurs flux financiers dans le long terme, d'étendre leurs sphères d'activité afin de contrôler l'ensemble de la chaîne de valeur de leur prestation, ou encore de trouver les moyens de financer des investissements initiaux souvent conséquents. Mais surtout elle requiert d'abandonner résolument un modèle d'affaires reposant sur la maximisation des ventes 
de produits, la coexistence d'activités aux objectifs diamétralement opposés ne pouvant que conduire tôt ou tard à des conflits d'intérêts délétères.

La mise en œuvre des principes de l'économie de fonctionnalité constitue l'une des rares voies permettant de conduire à une réduction non négligeable des pressions environnementales associées à la satisfaction d'une assez large gamme des besoins humains, sans que cela implique forcément d'abandonner un mode de développement centré sur la croissance économique. Cela ne veut pas dire pour autant qu'il n'est pas nécessaire d'effectuer des réformes dans la façon dont nous concevons nos rapports à l'environnement, et à autrui, si l'on veut qu'elle puisse développer tout son potentiel en tant que vecteur de durabilité. Les gains environnementaux qu'elle permet de réaliser sont ainsi bien trop souvent limités, du fait qu'elle n'est la plupart du temps envisagée que dans une optique purement économique.

De même, les initiatives visant à la mutualisation des biens et des services restent très insuffisamment développées, alors qu'elles sont riches de potentialités tant en termes de réduction des pressions environnementales que de tissage de liens sociaux et communautaires. Il serait ainsi à notre sens souhaitable que les réflexions futures sur l'économie de fonctionnalité - et d'une façon plus générale toutes celles qui ont pour objectif l'amélioration des capabilités humaines - cessent de n'envisager celle-ci qu'au travers du prisme de la technologie et du marché, et prennent davantage en compte le rôle que peuvent jouer le partage et la mutualisation dans la construction d'une société plus sobre et plus conviviale.

\section{BIBLIOGRAPHIE}

ADETEC, 2009, L'autopartage dans la sphère privée, étude réalisée pour le compte de l'ADEME et du MEEDDM dans le cadre du PREDIT (GO 3), www.adetec-deplacements.com/autopartage.pdf, consulté le 15 avril 2012.

Beuren F.H., Gomes Ferreira M.G., Cauchick Miguel P.A., 2013, "Product-service systems: a literature review on integrated products and services", Journal of Cleaner Production, vol. 47, p. 222-231.

Boulanger P.-M., 2007, « Une vérité qui dérange (certains) : on a encore besoin de l'écologie politique ! ", Etopia. Revue d'écologie politique, n 3, p. 31-61, http://etopia.les-verts.be/IMG/pdf/ revue_etopia_3.pdf, consulté le 15 avril 2012.

Bourg D., Buclet N., 2005, «L'économie de fonctionnalité : changer la consommation dans le sens du développement durable », Futuribles, n 313, p. 27-37.

Brezet J. C., Bijma A.S., Ehrenfeld J., Silvester S., 2001, The Design of Eco-Efficient Services. Method, Tools and Review of The Case Study Based "Designing Eco-Efficient Services" Project, Ministry of VROM, Delft University of Technology, Delft.

Caillé A., 2005, Don, intérêt et désintéressement - Bourdieu, Mauss, Platon et quelques autres, Nouvelle édition augmentée, Paris, La découverte, Mauss. 
Cooper, T., Evans S., 2000, Products to services, a report for Friends of the Earth, Sheffield Hallam University, The Centre for Sustainable Consumption, Sheffield.

Du Tertre C., 2011, « Modèles économiques d'entreprise, dynamique macroéconomique et développement durable », in Gaglio G., Lauriol J., du Tertre C. (dir.), L'économie de la fonctionnalité : une voie nouvelle vers un développement durable?, Toulouse, Octarès, p. 21-42.

Fandel M.-H., Zuleeg F., 2008, Gain without pain: towards a more rational use of energy, EPC Working Paper $N^{\circ}$ 29, European Policy Centre, Brussels.

Fishbein B. K., McGarry L. S., Dillon P. S., 2000, Leasing: A Step Toward Producer Responsibility, New York, Inform Inc.

Fishbein B. K., 2000, “Carpet Take-Back: EPR American Style”, Environmental Quality Management, vol. $10, \mathrm{n}^{\circ} 1$, p. $25-36$.

Gadrey J., 2003, Socio-économie des services, 3e édition, Paris, La Découverte, Collection Repères, $\mathrm{n}^{\circ}$ 369.

Goedkoop M.J., van Halen C.J.G., te Riele H.R.M., Rommens P.J.M., 1999, Product Service Systems: Ecological and Economic Basis, Report for Dutch Ministries of Environment (VROM) and Economic Affairs (EZ), The Netherlands.

Haefeli U., Matti D., Schreyer C., Maibach M., 2006, Evaluation Car-Sharing, Bundesamt für Energie, Bern.

Leblanc N., 2011, « La monnaie, nouvel espace de démocratie ? », Territoires, n515, http:// www.adels.org/territoires/515.htm, consulté le 15 avril 2012.

Lecocq X., Demil B., Warnier V., 2006, « Le business model, un outil d'analyse stratégique », L'Expansion Management Review, ${ }^{\circ}$ 123, p. 96-109.

Lindahl M., Ölundh G., 2001, “The Meaning of Functional Sales”, 8th International Seminar on Life Cycle Engineering, June 18-20, CIRP, Varna, Bulgaria.

Millard-Ball A., 2005, Car-Sharing: Where and How It Succeeds, TCRP Report 108, Transportation Research Board of the National Academies, Washington, DC, USA.

Moati P., 2008, L'économie des bouquets. Les marchés de solutions dans le nouveau capitalisme, La Tour d'Aigues, éditions de l'Aube.

Moati P., 2009, «Une économie des effets utiles », in Blondel C. et al., Vers un autre monde économique : dépression ou émergence?, Paris, Descartes \& Cie, Collection Forum d'action modernités, p. 149-167.

Mont O., 2002, "Clarifying the Concept of Product-Service System", Journal of Cleaner Production, vol. 10, Issue 3, p. 237-245.

Mont O., 2004, Product-service systems: Panacea or myth?, Doctoral dissertation, International Institute for Industrial Environmental Economics, Lund University, Lund, Sweden.

Polanyi K., 2008, Essais, Textes réunis et présentés par Michèle Cangiani et Jérôme Maucourant, Paris, Seuil, Collection Économie humaine.

Rifkin J., 2005, L'âge de l'accès. La nouvelle culture du capitalisme, trad. M. Saint-Upéry, 2e éd., Paris : La Découverte, (ed originale 2000).

Roy R., 2000, “Sustainable Product Service Systems”, Futures, Volume 32, Issue 3-4, p. 289-299.

Scott J. T., 2009, Managing the New Frontiers - An Introduction to the Fundamentals, Panama City, Management Education Services. 
Seid M. H., 2011, “Corporate Culture - DuPont becomes a franchisor”, MSA Worldwide, http:// www.msaworldwide.com/franchising-resources/articles/

Corporate_Culture_DuPont_becomes_a_franchisor, consulté le 15 avril 2012.

Stahel W., Giarini O., 1989, The Limits to Certainty: Facing Risks in the New Service Economy, Dordrecht, Kluwer Academic Publishers.

Stahel W., 1994, “The Utilization-Focused Service Economy”, in Allenby, B. R. (Ed.), The Greening of Industrial Ecosystems, Washington, DC, National Academy Press, p. 178-190.

Stahel W., 1997, “The Functional Economy: Cultural and Organizational Change”, in Richards D. J. (Ed.), The Industrial Green Game: Implications for Environmental Design and Management, Washington DC, National Academy Press, p. 91-100.

Stahel W., 2003, "Short list of successes, open verdicts and failures", prepared for the presentation on 'selling performance instead of goods' in Tokyo, September 2003, Geneva, Product-Life Institute.

Stahel W., 2006, The Performance Economy, London, Palgrave Macmillan.

Tukker A., Tischner U. (Eds.), 2004, New business for old Europe: Product-service development as a means to enhance competitiveness and eco-efficiency, final report of Suspronet, TNO-STB, Delft.

White A. L., Stoughton M., Feng L., 1999, Servicizing: The Quiet Transition to Extended Product Responsibility, Tellus Institute, Boston.

Zaring O., Bartolomeo M., Eder P., Hopkinson P., Groenewegen P., James P., de Jong P., Nijhuis L., Scholl G., Slob A., Örninge M., 2001, Creating Eco-efficient Producer Services, Gothenburg Research Institute, Gothenburg.

Zhao D., 2010, "Carsharing: A Sustainable and Innovative Personal Transport Solution with Great Potential and Huge Opportunities", www.frost.com/prod/servlet/market-insight-top.pag? Src=RSS\&docid=190795176, consulté le 15 avril 2012.

\section{NOTES}

1. Notons que ces auteurs ont proposé une définition des ventes fonctionnelles suffisamment large pour englober des offres de solutions telles que les services orientés résultat, les services orientés usage, les offres d'extension de la durée de vie des produits, ou encore de gestion de la demande. Nous restreignons pour notre part cette notion à un type bien particulier de vente d'usage, c'est-à-dire une offre dans laquelle le produit reste l'objet central de la transaction, fait l'objet d'un transfert de possession, et est manipulé par le client durant sa phase d'utilisation (voir plus bas).

2. Il existe un grand nombre de définitions rattachées au concept de PSS, qui a fait l'objet de plusieurs programmes de recherche. Nous avons choisi de retenir celle proposée par l'équipe d'Arnold Tukker dans le cadre du projet européen SusProNet, parce qu'elle procède d'une synthèse de recherches antérieures.

3. Alternatives Economiques $n^{\circ} 294$, septembre 2010, «L'économie de fonctionnalité, qu'est-ce que c'est? », www.alternatives-economiques.fr/l-economie-de-fonctionnalite--qu-est-ce-que-cest_fr_art_946_50214.html, consulté le 15 avril 2012.

4. Notons que plusieurs entreprises, comme le fabricant de téléphones Doro et le fabricant d'équipements de reproduction numérique et d'impression Kyocera Document Solutions Inc., se sont déjà emparées du terme "éco-fonctionnalité » pour désigner des programmes visant à réduire l'impact de leurs produits sur l'environnement. 
5. À l'image par exemple du concept de " développement durable », cet oxymore à l'allure pourtant si engageante qui, à force de répétitions et d'usages abusifs, s'est progressivement vidé de son sens et en qui beaucoup ne voient désormais plus qu'un terme galvaudé utilisé pour relayer le mythe de la perpétuation de la croissance.

6. Bien qu'il n'en soit pas absent à proprement parler, y étant de fait rangé dans la catégorie des PSS « orientés usage ».

7. Mobility, 2011, Rapport d'activité et de durabilité 2010, www.mobility.ch/files/pdf1/ Rappot_dactivite_et_durabilite_2010.pdf, consulté le 15 avril 2012.

8. Syndicat Mixte Autolib', 2010, «Autolib', une réalité », Conférence de presse du 16 décembre 2010, http://www.autolib-paris.fr/IMG/pdf/Dossier_de_presse_16_12_2010.pdf, consulté le 15 avril 2012.

9. Zipcar, 2012, "Zipcar Expands Car Sharing to San Jose, CA", press release, http:// zipcar.mediaroom.com/index.php?s=43\&item=268, consulté le 20 avril 2012.

10. Tant que nous sommes dans les services tournant autour de l'entretien de textiles, notons que l'exemple d'Elis, société française proposant un service de location-entretien de linge pour les professionnels (entreprises industrielles, hôtels...), n'est pas un exemple de consommation collective mais une offre de fonctionnalité « orientée résultat ». Les blanchisseries traditionnelles sont pour leur part des services « purs ».

11. InterfaceFLOR, 2010, "Avancée significative dans le recyclage de la moquette modulaire ", http://www.interfaceflor.com.cn/Internet/otherfiles.nsf/Lookup/FR_Press_ReEntry_May2010/ \$file/FR_Press_ReEntry_May2010.pdf, consulté le 15 avril 2012.

12. InterfaceFLOR, 2012, « Nos résultats », http://www.interfaceflor.fr/web/fr/developpement_ durable/mission_zero/nos_resultats, consulté le 29 septembre 2012.

13. Entretien personnel de l'auteur avec A. Salmon, directeur Systèmes et Finance de Lokéo, 23 avril 2012.

14. Lokéo, 2011, «En un an, Lokéo a su étendre le marché de la location à de nouveaux secteurs ", http://www.didiergras.com/espclient/fichiers/element/3149/LOKEO_1an.pdf, consulté le 15 avril 2012.

15. Rappelons que les machines à laver entrent dans cette catégorie de produits dont la nocivité envers l'environnement s'exprime majoritairement lors de leur utilisation, et non lors de leur fabrication ou de leur fin de vie.

16. Le contrat de performance énergétique (CPE) a été officiellement introduit en France par la loi de programmation relative à la mise en œuvre du Grenelle de l'Environnement (loi $\mathrm{n}^{\circ}$ 2009-967 du 3 août 2009), dite « loi Grenelle 1 ». Cet instrument financier, destiné à l'origine aux bâtiments publics (qui ont pour objectif de réduire de $40 \%$ leurs consommations énergétiques d'ici 2020), commence à être appliqué au secteur privé, où il pourrait permettre d'apporter une réponse à la problématique du financement des projets d'amélioration de la performance énergétique du parc immobilier ancien.

17. Berliner Energie Agentur, 2012, Energy Service Contracting, www.berliner-e-agentur.de/en/ topics/energy-service-contracting, consulté le 15 avril 2012.

18. Safechem, 2011, "Complease - Complete Process leasing - Individual package solution for optimized high precision cleaning while respecting the environment", The Dow Chemical Company, http://www.dow.com/safechem/pdf/773-00929.pdf, consulté le 15 avril 2012. 


\section{RÉSUMÉS}

L'économie de fonctionnalité consiste en la substitution de l'accès à une fonction d'usage à l'appropriation privative d'un bien matériel. Cet article a pour objet d'effectuer une présentation succincte des principes théoriques de ce concept et de la terminologie employée dans la littérature anglophone pour désigner les notions qui en sont dérivées. Une typologie des modèles de revenu d'entreprise au travers desquels il se décline est ensuite proposée: consommation collective, ventes de fonctions d'usage et contractualisation au résultat. Les mécanismes de ces modèles sont ensuite décrits et illustrés, et leurs avantages d'un point de vue environnemental discutés.

The functional service economy consists in substituting the access to a function to the private ownership of a product. This article aims at presenting the theoretical principles of this business model and the terminology used in the English-speaking literature to name the concepts that have been derived from it. A typology of the business models through which it is implemented is then proposed: collective consumption, use-oriented services and result-oriented services. The mechanisms of the models referenced in these three categories are then described and illustrated with examples of practical applications.

\section{INDEX}

Keywords : functional service economy, definition, terminology, business model, sustainable development

Mots-clés : économie de fonctionnalité, terminologie, modèle de revenu, développement durable

\section{AUTEUR}

\section{JOHAN VAN NIEL}

Johan Van Niel effectue une thèse de doctorat en co-tutelle à Université de Technologie de Troyes (UTT) et à Université de Lausanne (UNIL). La thèse est financée par l'ADEME et EDF. Johan_vanniel@yahoo.fr 